\title{
Unbalanced Translocation
}

National Cancer Institute

\section{Source}

National Cancer Institute. Unbalanced Translocation. NCI Thesaurus. Code C6823.

Interchanges of genetic material among different chromosomes following the breaking off of pieces of chromosomes such that the total chromosome composition does not contain all of the genetic material. 